\title{
The Global Existence of Solutions in Time for a Chemotaxis Model with Two Chemicals
}

\author{
Qian Xu, ${ }^{1}$ Xiaolin Liu, ${ }^{2}$ and Li Zhang ${ }^{1}$ \\ ${ }^{1}$ Department of Basic Courses, Beijing Union University, Beijing 100101, China \\ ${ }^{2}$ School of Sciences, Zhejiang A\&F University, Hangzhou, Zhejiang 311300, China \\ Correspondence should be addressed to Qian Xu; xuqian098@163.com
}

Received 6 April 2014; Revised 21 May 2014; Accepted 27 May 2014; Published 16 June 2014

Academic Editor: Zhidong Teng

Copyright (C) 2014 Qian Xu et al. This is an open access article distributed under the Creative Commons Attribution License, which permits unrestricted use, distribution, and reproduction in any medium, provided the original work is properly cited.

This paper concerns the uniform boundedness and global existence of solutions in time for the chemotaxis model with two chemicals. We prove the system has global existence of solutions in time for any dimension $n$.

\section{Introduction and Statement of Main Result}

Chemotaxis is the influence of chemical substances in the environment on the movement of mobile species. Keller and Segel [1] proposed the general chemotaxis system

$$
\begin{aligned}
& u_{t}=\nabla \cdot\left(D_{1}(u, v) \nabla u-D_{2}(u, v) \nabla v\right), \\
& v_{t}=D_{v} \Delta v-k(v) v+f(u, v),
\end{aligned}
$$

where $u$ is the density function of cells (e.g., Dictyostelium discoideum) that are attracted by a chemical substance (e.g., cAMP) produced by themselves and the movement towards a higher concentration of the chemical substance, whose concentration function is $v . D_{1}$ and $D_{v}$ are the random diffusion rates of cells and the chemical, respectively; $D_{2}(u, v) \nabla v$ represents the chemotactic flux of cells and $D_{2}(u, v)$ is positive for positive $u$ and $v$ and is called the sensitivity function; and $f(u, v)$ is the creation rate of the chemical, while $k(v) v$ is the degradation rate of the chemical.

The simplest case of (1) is that $D_{1}, D_{v}$, and $k$ are all positive constants, $f(u, v)=v$, and $D_{2}(u, v)=\chi u$ with $\chi$ being a positive constant. This was called by Childress and Perkus the "minimal model." When dimension $n=1$, solutions exist globally; see [2]. For $n=2$, global existence depends on a threshold: when the initial mass lies below the threshold solutions exist globally, while above the threshold solutions blow up in finite time; these results were derived by various authors; see the review article [3, 4]. Many authors have analyzed system (1) for several variants, such as global existence, blow up solutions, and many other results; see [3-5].

Painter et al. [6] proposed a chemotaxis model with two chemicals. They considered a Turing system [7] as a mechanism for providing spatially heterogeneous chemical distributions to which a cell population chemotactically responds. That was the following model:

$$
\begin{aligned}
& \frac{\partial w}{\partial t}=\nabla\left[D_{w} \nabla w-w \chi_{1}(u, v) \nabla u-w \chi_{2}(u, v) \nabla v\right], \\
& x \in \Omega, t>0, \\
& \frac{\partial u}{\partial t}=\nabla\left(D_{u} \cdot \nabla u\right)+f(u, v), \quad x \in \Omega, t>0, \\
& \frac{\partial v}{\partial t}=\nabla\left(D_{v} \cdot \nabla v\right)+g(u, v), \quad x \in \Omega, t>0, \\
& \frac{\partial w}{\partial v}=\frac{\partial u}{\partial v}=\frac{\partial v}{\partial v}=0, \quad x \in \partial \Omega, \\
& w(x, 0)=w_{0}(x), \quad u(x, 0)=u_{0}(x), \\
& v(x, 0)=v_{0}(x),
\end{aligned}
$$

where $w$ is a cell population, $u$ and $v$ are chemicals, and the cell population responds chemotactically to both chemical species. $\chi_{1}$ and $\chi_{2}$ are the chemotactic sensitivity functions and $f$ and $g$ define the chemical kinetics. $D_{w}, D_{u}$, and $D_{v}$ are taken as constants. 
A special form of system (2) is as follows:

$$
\begin{aligned}
& \frac{\partial w}{\partial t}=D \nabla\left(\nabla w-\frac{w}{\left(k_{1}+u\right)^{2}} \nabla u-\frac{w}{\left(k_{2}+v\right)^{2}} \nabla v\right), \\
& x \in \Omega, t>0, \\
& \frac{\partial u}{\partial t}=\Delta u+\delta-K u-u v^{2}, \quad x \in \Omega, t>0, \\
& \frac{\partial v}{\partial t}=\Delta v+K u+u v^{2}-v, \quad x \in \Omega, t>0 \\
& \frac{\partial w}{\partial v}=\frac{\partial u}{\partial v}=\frac{\partial v}{\partial v}=0, \quad x \in \partial \Omega, t>0, \\
& w(x, 0)=w_{0}(x) \geq 0, \quad u(x, 0)=u_{0}(x) \geq 0 \\
& v(x, 0)=v_{0}(x) \geq 0, \quad
\end{aligned}
$$

where $\Omega \in \mathbb{R}^{n}$ is a bounded domain. The kinetics of (3) are described by a simplified model for the glycolysis reaction $[8,9]$. As we know, the global existence of solutions in time of two species such as (1) is investigated by many authors; however, the global existence of solutions in time of three species is studied little. In this paper, we study the global existence of solutions in time of (3); by applying analysis semigroup and energy method we will prove that system (3) has global solutions in time in any dimension $n$.

We state the main result of this paper as follows.

Theorem 1. For any $w_{0}, u_{0}, v_{0} \in W^{1, p}(\Omega), p>n$ satisfying $w_{0} \geq 0, u_{0} \geq 0, v_{0} \geq 0$ on $\Omega$, (3) has a unique positive global solution $(w, u, v)$ such that

$$
\begin{gathered}
(w, u, v) \in C([0,+\infty), \\
\left.\quad W^{1, p}(\Omega) \times W^{1, p}(\Omega) \times W^{1, p}(\Omega)\right) \\
\cap C_{\text {loc }}^{2+2 \epsilon, 1+\epsilon}(\Omega \times(0,+\infty)) .
\end{gathered}
$$

\section{The Proof of Theorem 1}

Theorem 2. For any $w_{0}, u_{0}, v_{0} \in W^{1, p}(\Omega), p>n$ satisfying $w_{0} \geq 0, u_{0} \geq 0, v_{0} \geq 0$ on $\Omega$, one has the following conclusions.

(i) (3) has a unique solution $(w(x, t), u(x, t), v(x, t))$ on $\Omega \times\left[0, T_{\left(u_{0}, v_{0}\right)}\right)$ with $0<T_{\left(u_{0}, v_{0}\right)} \leq \infty$ satisfying

$$
\begin{aligned}
(w(\cdot, t), u(\cdot, t), v(\cdot, t)) \in C\left(\left[0, T_{\left(w_{0}, u_{0}, v_{0}\right)}\right),\right. & \\
& W^{1, p}(\Omega) \times W^{1, p}(\Omega) \\
& \left.\times W^{1, p}(\Omega)\right) \\
\cap C_{\text {loc }}^{2+2 \epsilon, 1+\epsilon}\left(\Omega \times\left(0, T_{\left(w_{0}, u_{0}, v_{0}\right)}\right)\right), & \\
& \text { for any } 0<\epsilon<\frac{1}{4} .
\end{aligned}
$$

(ii) Moreover, if, for small $\delta>0, t \in\left[\delta, T_{\left(w_{0}, u_{0}, v_{0}\right)}\right)$, $\|(w, u, v)(\cdot, t)\|_{L^{\infty}(\Omega)}$ is bounded; then $T_{\left(w_{0}, u_{0}, v_{0}\right)}=\infty$; that is, $(w, u, v)$ has global existence, and for any $0 \leq$ $\rho \leq \sigma \leq 1,(w, u, v) \in C^{\rho}\left([\delta, \infty), C^{2(1-\sigma)}(\Omega) \times\right.$ $\left.C^{2(1-\sigma)}(\Omega) \times C^{2(1-\sigma)}(\Omega)\right)$.

Proof. Equation (3) can be written as

$$
\begin{aligned}
& \left(\begin{array}{l}
w \\
u \\
v
\end{array}\right)_{t}=\nabla\left[A(u, v) \nabla\left(\begin{array}{l}
w \\
u \\
v
\end{array}\right)\right]+\left(\begin{array}{c}
0 \\
\delta-K u-u v^{2} \\
K u+u v^{2}-v
\end{array}\right), \\
& x \in \Omega, t>0, \\
& A(u, v) \nabla\left(\begin{array}{l}
w \\
u \\
v
\end{array}\right)=0, \quad x \in \partial \Omega, t>0,
\end{aligned}
$$

where

$$
A(u, v)=\left(\begin{array}{ccc}
D & -\frac{w}{\left(k_{1}+u\right)^{2}} & -\frac{w}{\left(k_{2}+v\right)^{2}} \\
0 & 1 & 0 \\
0 & 0 & 1
\end{array}\right)
$$

Since the eigenvalues of $A$ are positive, (6) is normally parabolic; then (i) follows from [10]. Note that (6) is also a "triangular system," so in virtue of [11], we complete the proof of Theorem 2.

In the following, we always assume $w_{0}, u_{0}, v_{0} \in$ $W^{1, p}(\Omega), p>n$ satisfying $w_{0} \geq 0, u_{0} \geq 0, v_{0} \geq 0$ on $\Omega$.

Lemma 3. For any dimension $n$, any solution $u$ of (3) has the following estimate:

$$
\|u\|_{L^{2}(\Omega)} \leq \mu_{1}, \quad 0 \leq t<T,
$$

where $T=T_{\left(w_{0}, u_{0}, v_{0}\right)}$ and $\mu_{1}$ depends only on $\left\|u_{0}\right\|_{L^{1}(\Omega)}$, $\left\|u_{0}\right\|_{L^{2}(\Omega)}, \delta, k$ and $|\Omega|$.

Proof. Integrating the second equation of (3) over $\Omega$, we have

$$
\begin{aligned}
\frac{d}{d t} \int_{\Omega} u d x & =\delta|\Omega|-K \int_{\Omega} u d x-\int_{\Omega} u v^{2} d x \\
& \leq-K \int_{\Omega} u d x+\delta|\Omega| .
\end{aligned}
$$

Integrating (9) with respect to $t$, we get

$$
\begin{aligned}
& \int_{\Omega} u d x \leq e^{-K t}\left\|u_{0}\right\|_{L^{1}(\Omega)}+\frac{\delta|\Omega|}{K}, \\
& \text { that is }\|u\|_{L^{1}(\Omega)} \leq C, \quad C \sim\left\|u_{0}\right\|_{L^{1}(\Omega)}, \quad \delta, K,|\Omega| .
\end{aligned}
$$

Multiplying the second equation of (3) by $u$ and integrating with respect to $x$ over $\Omega$, we get

$$
\begin{aligned}
\frac{d}{d t} \int_{\Omega} u^{2} d x= & -2 \int_{\Omega}|\nabla u|^{2} d x+2 \delta \int_{\Omega} u d x \\
& -2 K \int_{\Omega} u^{2} d x-2 \int_{\Omega} u^{2} v^{2} d x \\
\leq & -2 K \int_{\Omega} u^{2} d x+2 \delta\|u\|_{L^{1}(\Omega)} .
\end{aligned}
$$


Integrating (11) with respect to $t$ and together with (10), we obtain

$$
\|u\|_{L^{2}(\Omega)} \leq \mu_{1}, \quad \mu_{1} \sim\left\|u_{0}\right\|_{L^{1}(\Omega)}, \quad\left\|u_{0}\right\|_{L^{2}(\Omega)}, \quad \delta, K,|\Omega| .
$$

Lemma 4. For any dimension $n$ and small constants $\tau_{0}>0$, any solution $u$ of (3) has the following estimate:

$$
\|u\|_{L^{\infty}(\Omega)} \leq \mu_{2}, \quad \tau_{0} \leq t<T,
$$

where $T=T_{\left(w_{0}, u_{0}, v_{0}\right)}$ and $\mu_{2}$ is a constant depending on $\left\|u_{0}\right\|_{L^{1}(\Omega)},\left\|u_{0}\right\|_{L^{2}(\Omega)}, \delta, k,|\Omega|$ and $\left\|u\left(\cdot, \tau_{0}\right)\right\|_{L^{\infty}(\Omega)}$.

Proof. Multiplying the second equation of (3) by $u^{p-1}(p \geq 2)$ and integrating with respect to $x$ over $\Omega$, we get

$$
\begin{aligned}
\frac{1}{p} \frac{d}{d t} \int_{\Omega} u^{p} d x= & -\frac{4(p-1)}{p^{2}} \int_{\Omega}\left|\nabla\left(u^{p / 2}\right)\right|^{2} d x \\
& +\delta \int_{\Omega} u^{p-1} d x-K \int_{\Omega} u^{p} d x-\int_{\Omega} u^{p} v^{2} d x .
\end{aligned}
$$

Here (14) implies

$$
\begin{aligned}
& \frac{d}{d t} \int_{\Omega} u^{p} d x+\frac{4(p-1)}{p} \int_{\Omega}\left|\nabla\left(u^{p / 2}\right)\right|^{2} d x \\
& \quad \leq \delta p \int_{\Omega} u^{p-1} d x-K p \int_{\Omega} u^{p} d x .
\end{aligned}
$$

Define $z=u^{p / 2}$, by Hölder's inequality; (15) can be written as

$$
\begin{aligned}
& \frac{d}{d t} \int_{\Omega} z^{2} d x+\frac{4(p-1)}{p} \int_{\Omega}|\nabla z|^{2} d x \\
& \quad \leq \delta p \int_{\Omega} z^{2(p-1) / p} d x-K p \int_{\Omega} z^{2} d x \\
& \quad \leq \delta p|\Omega|^{1 / p}\|z\|_{L^{2}}^{2(p-1) / p} .
\end{aligned}
$$

Gagliardo-Nirenberg inequality implies

$$
\begin{aligned}
\|z\|_{L^{2}(\Omega)} \leq & C_{1}\|\nabla z\|_{L^{2}(\Omega)}^{\theta}\|z\|_{L^{1}(\Omega)}^{1-\theta} \\
& +C_{2}\|z\|_{L^{1}(\Omega)}, \quad \text { with } \theta=\frac{n}{n+2} .
\end{aligned}
$$

In virtue of (17) and Young's inequality, we have

$$
\begin{aligned}
\|z\|_{L^{2}}^{2(p-1) / p} \leq & \left(2 C_{1}^{2}\|\nabla z\|_{L^{2}(\Omega)}^{2 \theta}\|z\|_{L^{1}(\Omega)}^{2(1-\theta)}\right. \\
& \left.+2 C_{2}^{2}\|z\|_{L^{1}(\Omega)}^{2}\right) \\
\leq & 2^{(p-1) / p} C_{1}^{2(p-1) / p}\|\nabla z\|_{L^{2}(\Omega)}^{2(p-1) \theta / p} \\
& \times\|z\|_{L^{1}(\Omega)}^{2(p-1)(1-\theta) / p} \\
& +2^{(p-1) / p} C_{2}^{(2(p-1) / p)}\|z\|_{L^{1}(\Omega)}^{2(p-1) / p}
\end{aligned}
$$

$$
\begin{aligned}
\leq & \frac{(p-1) \theta}{p} \varepsilon^{p /(p-1) \theta}\|\nabla z\|_{L^{2}(\Omega)}^{2} \\
& +\frac{p-(p-1) \theta}{p} \varepsilon^{-p /(p-(p-1) \theta)} 2^{(p-1) /(p-(p-1) \theta)} \\
C_{1}^{2(p-1) /(p-(p-1) \theta)}\|z\|_{L^{1}(\Omega)}^{2(p-1)(1-\theta) /(p-(p-1) \theta)} & +2^{(p-1) / p} C_{2}^{2(p-1) / p}\|z\|_{L^{1}(\Omega)}^{2(p-1) / p} \\
\leq & \frac{(p-1) \theta}{p} \varepsilon^{p /(p-1) \theta}\|\nabla z\|_{L^{2}(\Omega)}^{2} \\
& +C_{3} \varepsilon^{-p /(p-(p-1) \theta)}\|z\|_{L^{1}(\Omega)}^{2(p-1)(1-\theta) /(p-(p-1) \theta)} \\
& +C_{4}\|z\|_{L^{1}(\Omega)}^{2(p-1) / p}
\end{aligned}
$$

with $C_{3}=2^{(n+2) / 2} C_{1}^{n+2}$ and $C_{4}=2 C_{2}^{2}$.

Taking suitable $\varepsilon$ such that $\delta p|\Omega|^{1 / p}((p-1) \theta / p) \varepsilon^{p /(p-1) \theta}=$ $3(p-1) / p$, we obtain

$$
\begin{aligned}
\varepsilon^{-p /(p-(p-1) \theta)} & =\left[\left(\frac{3}{\delta p|\Omega|^{1 / p} \theta}\right)^{(p-1) \theta / p}\right]^{-p /(p-(p-1) \theta)} \\
& <C_{5} p^{n / 2}
\end{aligned}
$$

with $C_{5}=\left(\delta|\Omega|^{1 / p} \theta / 3\right)^{n / 2}$.

In view of (16)-(19), we get

$$
\begin{aligned}
& \frac{d}{d t} \int_{\Omega} z^{2} d x+\frac{(p-1)}{p} \int_{\Omega}|\nabla z|^{2} d x \\
& \leq C_{3} C_{5} p^{(n+2) / 2} \delta|\Omega|^{1 / p}\|z\|_{L^{1}(\Omega)}^{2(p-1)(1-\theta) /(p-(p-1) \theta)} \\
& \quad+C_{4} \delta p|\Omega|^{(1 / p)}\|z\|_{L^{1}(\Omega)}^{2(p-1) / p} \\
& \leq C_{6} \delta p^{(n+2) / 2}\|z\|_{L^{1}(\Omega)}^{2(p-1)(1-\theta) /(p-(p-1) \theta)} \\
& \quad+C_{7} \delta p\|z\|_{L^{1}(\Omega)}^{2(p-1) / p}
\end{aligned}
$$

with $C_{6}=C_{3} C_{5}|\Omega|^{1 / p}$ and $C_{7}=C_{4}|\Omega|^{1 / p}$.

By Poincaré inequality, there exists a constant $\sigma>0$ depending on $n, p, \Omega$ such that

$$
\|z\|_{L^{2}(\Omega)}^{2} \leq \frac{1}{\sigma}\left(\|\nabla z\|_{L^{2}(\Omega)}^{2}+\|z\|_{L^{1}(\Omega)}^{2}\right) ;
$$

we have

$$
\frac{p-1}{p} \int_{\Omega}|\nabla z|^{2} d x \geq \frac{p-1}{p} \sigma \int_{\Omega} z^{2} d x-\frac{p-1}{p}\|z\|_{L^{1}(\Omega)}^{2} .
$$


It follows from (20) and (22) that

$$
\begin{aligned}
\frac{d}{d t} \int_{\Omega} z^{2} d x \leq & -\frac{p-1}{p} \sigma \int_{\Omega} z^{2} d x \\
& +C_{6} \delta p^{(n+2) / 2}\|z\|_{L^{1}(\Omega)}^{2(p-1)(1-\theta) /(p-(p-1) \theta)} \\
& +C_{7} \delta p\|z\|_{L^{1}(\Omega)}^{(2(p-1) / p)}+\frac{p-1}{p}\|z\|_{L^{1}(\Omega)}^{2} .
\end{aligned}
$$

After simple calculation, we obtain

$$
\begin{aligned}
\|z\|_{L^{2}(\Omega)}^{2} \leq \max \{ & C_{8} p^{(n+2) / 2} \sup _{t \geq \tau_{0}}\|z\|_{L^{1}(\Omega)}^{2(p-1)(1-\theta) /(p-(p-1) \theta)} \\
& +C_{9} p \sup _{t \geq \tau_{0}}\|z\|_{L^{1}(\Omega)}^{2(p-1) / p} \\
& \left.+C_{10} \sup _{t \geq \tau_{0}}\|z\|_{L^{1}(\Omega)}^{2},\left\|z\left(\tau_{0}\right)\right\|_{L^{2}(\Omega)}^{2}\right\}
\end{aligned}
$$

with $C_{8}=C_{6} \delta / \sigma, C_{9}=C_{7} \delta / \sigma$, and $C_{10}=1 / \sigma$.

Since $2(p-1)(1-\theta) /(p-(p-1) \theta)<2$ and $2(p-1) / p<2$, we have

$$
\begin{aligned}
& \sup _{t \geq \tau_{0}}\|z\|_{L^{2}(\Omega)}^{2} \leq \max \{ C_{11} p^{(n+2) / 2} \\
& \times \max \left\{\sup _{t \geq \tau_{0}}\|z\|_{L^{1}(\Omega)}^{2}, 1\right\}, \\
&\left.\left\|z\left(\tau_{0}\right)\right\|_{L^{2}(\Omega)}^{2}\right\},
\end{aligned}
$$

with $C_{11}=\max \left\{C_{8}, C_{9}, C_{10}\right\}$.

Substituting $z=u^{p / 2}, p=2^{k}, k=1,2,3, \ldots$ into (25) yields

$$
\begin{gathered}
\sup _{t \geq \tau_{0}} \int_{\Omega} u^{2^{k}} d x \\
\leq \max \left\{C_{11} 2^{((n+2) / 2) k} \max \left\{\left(\sup _{t \geq \tau_{0}} \int_{\Omega} u^{2^{k-1}} d x\right)^{2}, 1\right\},\right. \\
\left.\left\|u\left(\tau_{0}\right)\right\|_{L^{\infty}(\Omega)}^{2^{k}}\right\} .
\end{gathered}
$$

Without loss of generality, we can assume

$$
\sup _{t \geq \tau_{0}} \int_{\Omega} u^{2^{k}} d x \leq C_{11} 2^{((n+2) / 2) k}\left(\sup _{t \geq \tau_{0}} \int_{\Omega} u^{2^{k-1}} d x\right)^{2}
$$

$$
\begin{gathered}
\sup _{t \geq \tau_{0}} \int_{\Omega} u^{2^{k}} d x \leq\left[C_{11} 2^{((n+2) / 2) k}\right]^{2^{0}}\left[C_{11} 2^{((n+2) / 2)(k-1)}\right]^{2^{1}} \cdots \\
{\left[C_{11} 2^{((n+2) / 2)(k-(k-3))}\right]^{2^{k-3}},} \\
{\left[C_{11} 2^{((n+2) / 2)(k-(k-2))}\right]^{2^{k-2}}\left(\sup _{t \geq \tau_{0}} \int_{\Omega} u^{2^{k-(k-1)}} d x\right)^{2^{k-1}}} \\
\leq C_{11}^{2^{(k-1)}-1} \\
\times 2^{((n+2) / 2)\left(k+(k-1) 2^{1}+\cdots+4 \cdot 2^{k-4}+3 \cdot 2^{k-3}+2 \cdot 2^{k-2}+2^{k-1}\right)} \\
\times\left(\sup _{t \geq \tau_{0}} \int_{\Omega} u^{2} d x\right)^{2^{k-1}} .
\end{gathered}
$$

Taking $1 / 2^{k}$ and letting $k \rightarrow \infty$ of (28), together with Lemma 3, we have

$$
\sup _{t \geq \tau_{0}}\|u\|_{L^{\infty}(\Omega)} \leq C_{11}^{1 / 2} 2^{(n+2) / 2}\left(\sup _{t \geq \tau_{0}} \int_{\Omega} u^{2} d x\right)^{1 / 2} \leq \mu_{2} .
$$

With the notation

$$
\bar{w}=u+v,
$$

we have the following Lemma 5.

Lemma 5. For any dimension $n, \bar{w}$ has the following estimate:

$$
\|\bar{w}\|_{C^{1}(\Omega)} \leq \mu_{3}, \quad \tau_{0} \leq t<T,
$$

where $T=T_{\left(w_{0}, u_{0}, v_{0}\right)}$ and $\mu_{3}$ is a constant depending on $\left\|u_{0}\right\|_{L^{1}(\Omega)},\left\|u_{0}\right\|_{L^{2}(\Omega)}, \delta, \quad k, \quad|\Omega|,\left\|u\left(\cdot, \tau_{0}\right)\right\|_{L^{\infty}(\Omega)}$, and $\left\|\bar{w}\left(\cdot, \tau_{0}\right)\right\|_{W^{2, p}(\Omega)}$.

Proof. In view of (3), $\bar{w}$ satisfies the following equation:

$$
\frac{d \bar{w}}{d t}=\Delta \bar{w}+\delta+u-\bar{w}
$$

let $X=L^{p}(\Omega), A=\Delta-I$ with domain $D=W^{2, p}(\Omega)$; then $A$ generates a linear analysis semigroup on $X$ satisfying $\left\|e^{A t}\right\| \leq$ $C e^{-a t}$ and $\left\|e^{A t}\right\|_{X^{\alpha}} \leq C_{\alpha} t^{-\alpha} e^{-a t}$ for $0<a<1$. Taking $p>n$, the fractional space $X^{\alpha} \hookrightarrow C^{\gamma}$ with $0 \leq \gamma<2 \alpha-(n / p)$; taking $\alpha=(1 / 2)+(n / 2 p)$ we get $\gamma=1$.

In virtue of (32), we obtain

$$
\begin{gathered}
\bar{w}(\cdot, t)=e^{A\left(t-\tau_{0}\right)} \bar{w}\left(\cdot, \tau_{0}\right)+\int_{\tau_{0}}^{t} e^{A(t-s)}(\delta+u(\cdot, s)) d s, \\
\|\bar{w}(\cdot, t)\|_{C^{1}(\Omega)} \leq C_{1}\|\bar{w}(\cdot, t)\|_{X^{\alpha}} \\
\leq C_{1}\left\|e^{A\left(t-\tau_{0}\right)} \bar{w}\left(\cdot, \tau_{0}\right)\right\|_{X^{\alpha}} \\
+C_{1} \int_{\tau_{0}}^{t}\left\|e^{A(t-s)}(\delta+u(\cdot, s))\right\|_{X^{\alpha}} d s
\end{gathered}
$$




$$
\begin{aligned}
\leq & C_{2} e^{-a\left(t-\tau_{0}\right)}\left\|\bar{w}\left(\cdot, \tau_{0}\right)\right\|_{X^{\alpha}} \\
& +\int_{\tau_{0}}^{t} C_{\alpha}(t-s)^{-\alpha} e^{-a(t-s)}\|\delta+u(\cdot, s)\|_{L^{p}(\Omega)} d s \\
\leq & C_{3}+C_{4} \sup _{\tau_{0} \leq s \leq t}\|u(\cdot, t)\|_{L^{p}(\Omega)} \\
\leq & \mu_{3} .
\end{aligned}
$$

By Lemma 4 and (30), one has

$$
\|v\|_{L^{\infty}(\Omega)} \leq\|u\|_{L^{\infty}(\Omega)}+\|\bar{w}\|_{L^{\infty}(\Omega)} \leq \mu_{4},
$$

where $\mu_{4}$ is a constant depending on $\left\|u_{0}\right\|_{L^{1}(\Omega)},\left\|u_{0}\right\|_{L^{2}(\Omega)}, \delta$, $k,|\Omega|,\left\|u\left(\cdot, \tau_{0}\right)\right\|_{L^{\infty}(\Omega)}$, and $\left\|\bar{w}\left(\cdot, \tau_{0}\right)\right\|_{W^{2, p}}(\Omega)$.

Similar as the proof of Lemma 5, we can prove

$$
\|v\|_{C^{1}(\Omega)} \leq \mu_{5},
$$

where $\mu_{5}$ is a constant depending on $\left\|u_{0}\right\|_{L^{1}(\Omega)},\left\|u_{0}\right\|_{L^{2}(\Omega)}, \delta$, $k,|\Omega|,\left\|u\left(\cdot, \tau_{0}\right)\right\|_{L^{\infty}(\Omega)},\left\|\bar{w}\left(\cdot, \tau_{0}\right)\right\|_{W^{2, p}(\Omega)}$, and $\left\|v\left(\cdot, \tau_{0}\right)\right\|_{W^{2, p}(\Omega)}$.

Lemma 5 and (35) yield

$$
\|u\|_{C^{1}(\Omega)} \leq \mu_{6},
$$

where $\mu_{6}$ is a constant depending on $\left\|u_{0}\right\|_{L^{1}(\Omega)}$, $\left\|u_{0}\right\|_{L^{2}(\Omega)}, \delta, k,|\Omega|,\left\|u\left(\cdot, \tau_{0}\right)\right\|_{L^{\infty}(\Omega)},\left\|\bar{w}\left(\cdot, \tau_{0}\right)\right\|_{W^{2, p}(\Omega)}, \quad$ and $\left\|v\left(\cdot, \tau_{0}\right)\right\|_{W^{2, p}(\Omega)}$.

Lemma 6. For any dimension $n$, any solution $\omega$ of (3) has the following estimate:

$$
\|w\|_{L^{\infty}(\Omega)} \leq \mu_{7}, \quad \tau_{0} \leq t<T,
$$

where $T=T_{\left(w_{0}, u_{0}, v_{0}\right)}$ and $\mu_{7}$ is a constant depending on $\left\|u_{0}\right\|_{L^{1}(\Omega)},\left\|u_{0}\right\|_{L^{2}(\Omega)}, \delta, k,|\Omega|,\left\|u\left(\cdot, \tau_{0}\right)\right\|_{L^{\infty}(\Omega)},\left\|\bar{w}\left(\cdot, \tau_{0}\right)\right\|_{W^{2, p}(\Omega)}$, $\left\|v\left(\cdot, \tau_{0}\right)\right\|_{W^{2, p}(\Omega)},\left\|w_{0}(x)\right\|_{L^{1}(\Omega)}$, and $\left\|w\left(\cdot, \tau_{0}\right)\right\|_{L^{\infty}(\Omega)} \cdot$

Proof. Integrating the first equation of (3) with respect to $x$ over $\Omega$ and together with the boundary condition, we get

$$
\int_{\Omega} w(x, t) d x=\int_{\Omega} w_{0}(x) d x \leq C_{1} .
$$

In the following, we will use the inequality as follows:

$$
\|u\|_{L^{2}(\Omega)}^{2} \leq \varepsilon\|\nabla u\|_{L^{2}(\Omega)}^{2}+C\left(1+\varepsilon^{-n / 2}\right)\|u\|_{L^{1}(\Omega)}^{2},
$$

with $C$ depending only on $n$ and $\Omega$.

Multiplying the first equation of (3) by $w^{s-1}(s \geq 2)$ and integrating with respect to $x$ over $\Omega$ imply

$$
\begin{aligned}
\frac{1}{s} \frac{d}{d t} \int_{\Omega} w^{s} d x= & -D(s-1) \int_{\Omega} w^{s-2}|\nabla w|^{2} d x \\
& +(s-1) \int_{\Omega} \frac{w^{s-1}}{\left(k_{1}+u\right)^{2}} \nabla u \cdot \nabla w d x \\
& +(s-1) \int_{\Omega} \frac{w^{s-1}}{\left(k_{2}+v\right)^{2}} \nabla v \cdot \nabla w d x
\end{aligned}
$$

$$
\begin{aligned}
\leq & \frac{-4 D(s-1)}{s^{2}} \int_{\Omega}\left|\nabla\left(w^{s / 2}\right)\right|^{2} d x \\
& +C_{2}(s-1) \int_{\Omega} w^{s-1} \nabla w d x \\
& +C_{3}(s-1) \int_{\Omega} w^{s-1} \nabla w d x \\
\leq & \frac{-4 D(s-1)}{s^{2}} \int_{\Omega}\left|\nabla\left(w^{s / 2}\right)\right|^{2} d x \\
& +C_{4} \int_{\Omega} w^{s / 2} \nabla\left(w^{s / 2}\right) d x .
\end{aligned}
$$

(39) and Hölder's inequality yield

$$
\begin{aligned}
\frac{d}{d t} \int_{\Omega} w^{s} d x \leq & \frac{-4 D(s-1)}{s} \int_{\Omega}\left|\nabla\left(w^{s / 2}\right)\right|^{2} d x \\
& +C_{4} s\left(\int_{\Omega} w^{s} d x\right)^{1 / 2}\left(\int_{\Omega}\left|\nabla\left(w^{s / 2}\right)\right|^{2} d x\right)^{1 / 2} \\
\leq & -2 D \int_{\Omega}\left|\nabla\left(w^{s / 2}\right)\right|^{2} d x \\
& +D \int_{\Omega}\left|\nabla\left(w^{s / 2}\right)\right|^{2} d x+\frac{C_{4}^{2} s^{2}}{4 D} \int_{\Omega} w^{s} d x \\
\leq & -D \int_{\Omega}\left|\nabla\left(w^{s / 2}\right)\right|^{2} d x+C_{5} s^{2} \int_{\Omega} w^{s} d x \\
\leq & \frac{D\left(C \varepsilon^{-n / 2}+1\right)}{\varepsilon}\left(\int_{\Omega} w^{s / 2} d x\right)^{2} \\
& -\frac{D}{\varepsilon} \int_{\Omega} w^{s} d x+C_{5} s^{2} \int_{\Omega} w^{s} d x \\
\leq & -C_{5} s^{2} \int_{\Omega} w^{s} d x+C_{6} s^{n+2}\left(\int_{\Omega} w^{s / 2} d x\right)^{2} .
\end{aligned}
$$

For $\tau_{0} \leq t \leq T$, by (41), we have

$$
\frac{d}{d t}\left(e^{C_{5} s^{2} t} \int_{\Omega} w^{s} d x\right) \leq C_{6} s^{n+2} e^{C_{5} s^{2} t}\left(\int_{\Omega} w^{s / 2} d x\right)^{2} .
$$

Integrating (42) with respect to $t$ over $\left[\tau_{0}, t\right]$, we obtain

$$
\begin{aligned}
\int_{\Omega} w^{s}(x, t) d x \leq & e^{C_{5} s^{2}\left(\tau_{0}-t\right)} \int_{\Omega} w^{s}\left(x, \tau_{0}\right) d x \\
& +C_{7} s^{n}\left(1-e^{C_{5} s^{2}\left(\tau_{0}-t\right)}\right) \\
& \times \sup _{\tau_{0} \leq t \leq T}\left(\int_{\Omega} w^{s / 2} d x\right)^{2} \\
\leq & \left\|w\left(x, \tau_{0}\right)\right\|_{L^{\infty}(\Omega)}^{s} \\
& +C_{8} s^{n} \sup _{\tau_{0} \leq t \leq T}\left(\int_{\Omega} w^{s / 2} d x\right)^{2} .
\end{aligned}
$$




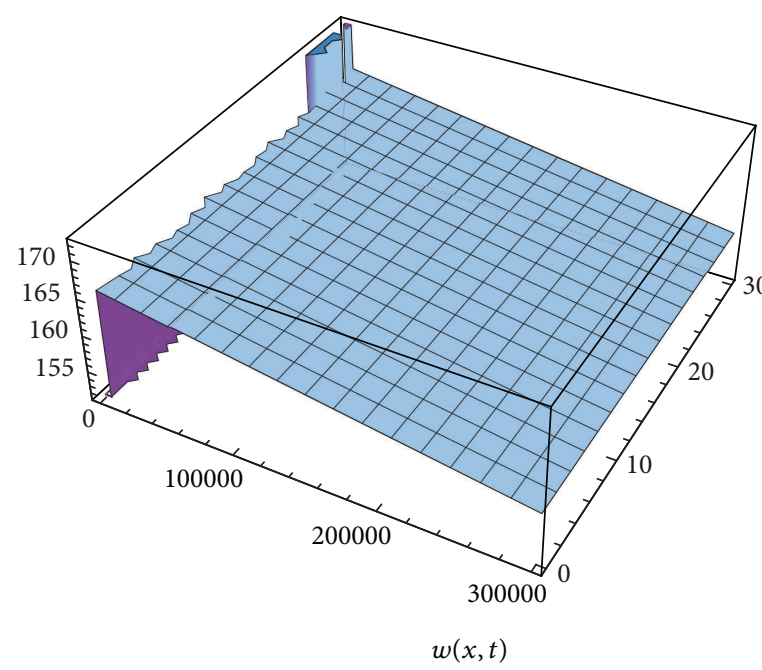

(a)

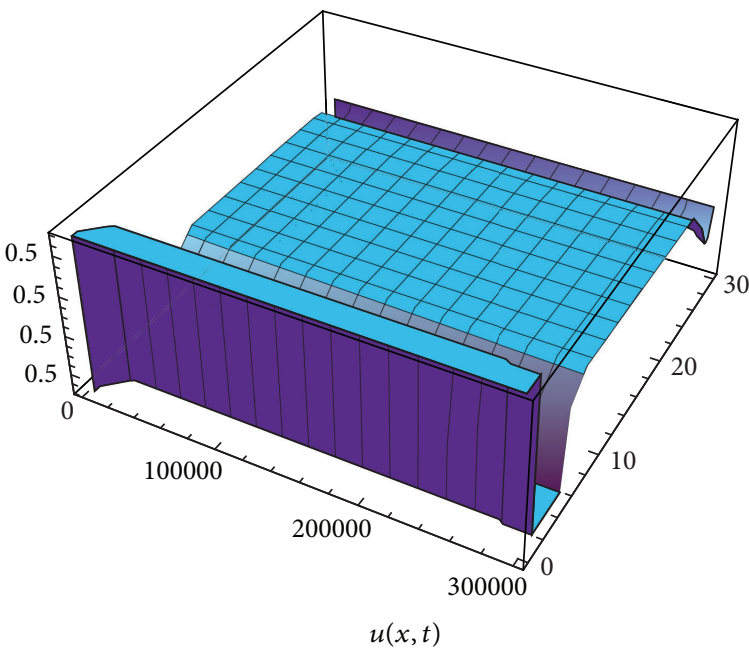

(b)

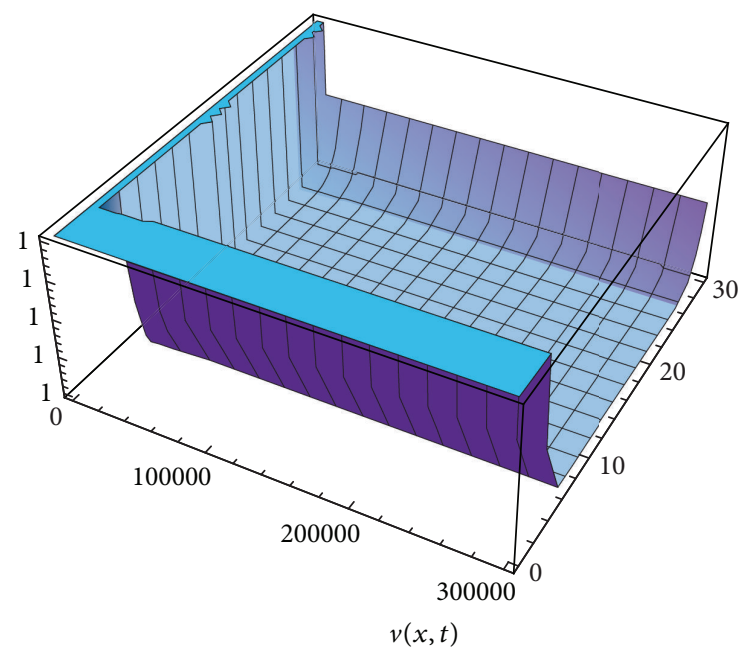

(c)

Figure 1

Let

$$
M(s)=\max \left\{\left\|w\left(x, \tau_{0}\right)\right\|_{L^{\infty}(\Omega)}, \sup _{\tau_{0} \leq t \leq T}\left(\int_{\Omega} w^{s} d x\right)^{1 / s}\right\}
$$

then we get

$$
M(s) \leq\left(C_{9} s^{n}\right)^{(1 / s)} M\left(\frac{s}{2}\right), \quad s \geq 2 .
$$

Taking $s=2^{k}, k=1,2, \ldots$, we get

$$
\begin{aligned}
M\left(2^{k}\right) & \leq C_{9}^{1 / 2^{k}} 2^{k n / 2^{k}} M\left(2^{k-1}\right) \\
& \leq C_{9}^{\left(1 / 2^{k}\right)+\cdots+(1 / 2)} 2^{\left(k n / 2^{k}\right)+\cdots+(n / 2)} M(1) \\
& \leq C M(1) .
\end{aligned}
$$

For $\tau_{0}<+\infty$ and (38) one gets

$$
\|w(\cdot, t)\|_{L^{\infty}(\Omega)} \leq \sup _{\tau_{0} \leq t \leq T} \int_{\Omega} w d x \leq \mu_{7},
$$

where $\mu_{7}$ is a constant depending on $\left\|u_{0}\right\|_{L^{1}(\Omega)}$, $\left\|u_{0}\right\|_{L^{2}(\Omega)}, \delta, \quad k, \quad|\Omega|,\left\|u\left(\cdot, \tau_{0}\right)\right\|_{L^{\infty}(\Omega)}, \quad\left\|\bar{w}\left(\cdot, \tau_{0}\right)\right\|_{W^{2, p}(\Omega)}$, $\left\|v\left(\cdot, \tau_{0}\right)\right\|_{W^{2, p}(\Omega)},\left\|w_{0}(x)\right\|_{L^{1}(\Omega)}$, and $\left\|w\left(\cdot, \tau_{0}\right)\right\|_{L^{\infty}(\Omega)}$. Now by Theorem 2(ii) and Lemma 3-Lemma 6, we have proved the Theorem 1.

For $D=k_{1}=k_{2}=\delta=K=1, T=300000, \Omega=[0,30]$, $w_{0}(x)=(1 / 10000) x^{3}(30-x)^{2}, u_{0}(x)=x^{3}(30-x)^{2}$, and $v_{0}(x)=x^{2}(30-x)^{2}$, we have the numerical simulation solutions of (3) as shown in Figure 1.

\section{Conflict of Interests}

The authors declare that there is no conflict of interests regarding the publication of this paper. 


\section{Acknowledgments}

This work is partially supported by the NNSF of China (71373023), the Beijing Natural Science Foundation (1132003, 1122016, KZ201310028030, KM201311417006, and KM201210017008), and the Zhejiang A\&F University Talent Program (2013FR078).

\section{References}

[1] E. Keller and L. Segel, "Initiation of slime mold aggregation viewed as an instability," Journal of Theoretical Biology, vol. 26, pp. 399-415, 1970.

[2] K. Osaki and A. Yagi, "Finite dimensional attractor for onedimensional Keller-Segel equations," Funkcialaj Ekvacioj, vol. 44, no. 3, pp. 441-469, 2001.

[3] D. Horstmann, "From 1970 until present: the Keller-Segel model in chemotaxis and its consequences. I," Jahresbericht der Deutschen Mathematiker-Vereinigung, vol. 105, pp. 103-165, 2003.

[4] D. Horstmann, "From 1970 until present: the Keller-Segel model in chemotaxis and its consequences. II," Jahresbericht der Deutschen Mathematiker-Vereinigung, vol. 106, no. 2, pp. 51-69, 2004.

[5] T. Hillen and K. J. Painter, "A user's guide to PDE models for chemotaxis," Journal of Mathematical Biology, vol. 58, no. 1-2, pp. 183-217, 2009.

[6] K. J. Painter, P. K. Maini, and H. G. Othmer, "Development and applications of a model for cellular response to multiple chemotactic cues," Journal of Mathematical Biology, vol. 41, no. 4, pp. 285-314, 2000.

[7] A. M. Turing, "The chemical basis for morphogenesis," Philosophical Transactions of the Royal Society of London B, vol. 237, pp. 37-72, 1952.

[8] R. Dillon, P. K. Maini, and H. G. Othmer, "Pattern formation in generalized Turing systems. I. Steady-state patterns in systems with mixed boundary conditions," Journal of Mathematical Biology, vol. 32, no. 4, pp. 345-393, 1994.

[9] H. G. Othmer and J. A. Aldridge, "The effects of cell density and metabolite flux on cellular dynamics," Journal of Mathematical Biology, vol. 5, no. 2, pp. 169-200, 1978.

[10] H. Amann, "Dynamic theory of quasilinear parabolic equations. II. Reaction-diffusion systems," Differential and Integral Equations, vol. 3, no. 1, pp. 13-75, 1990.

[11] H. Amann, "Dynamic theory of quasilinear parabolic systems. III. Global existence," Mathematische Zeitschrift, vol. 202, no. 2, pp. 219-250, 1989. 


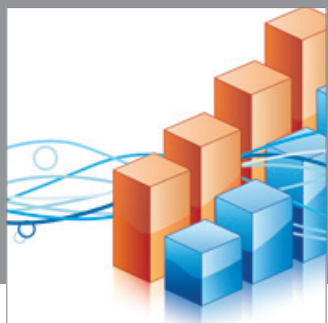

Advances in

Operations Research

mansans

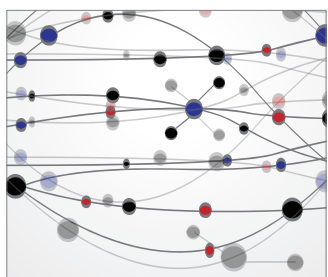

The Scientific World Journal
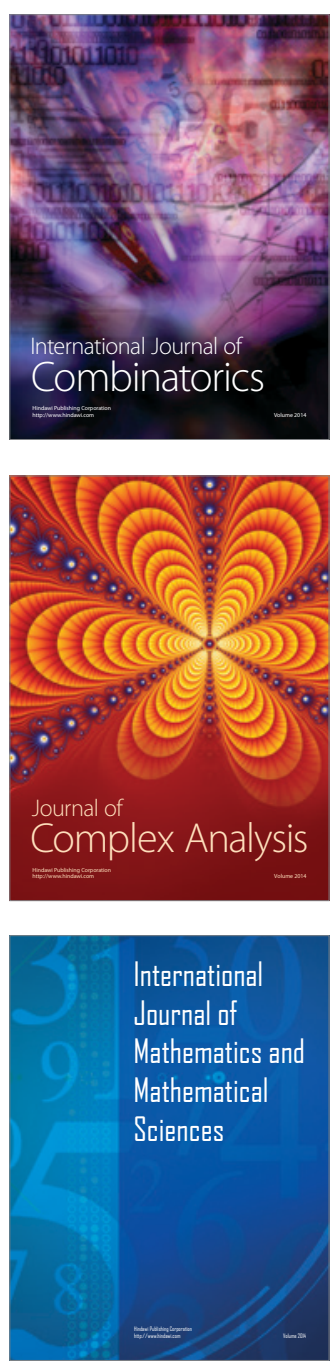
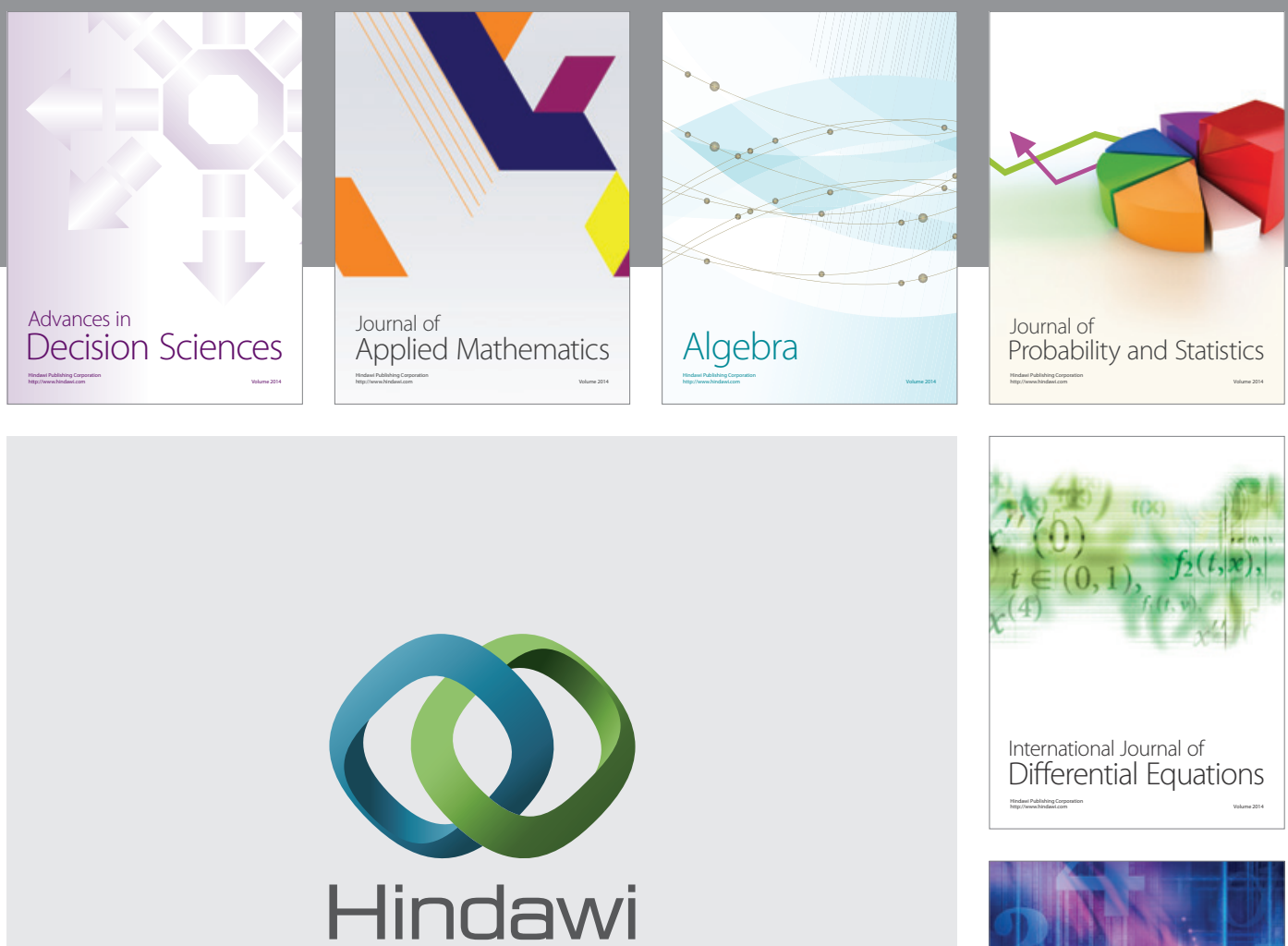

Submit your manuscripts at http://www.hindawi.com
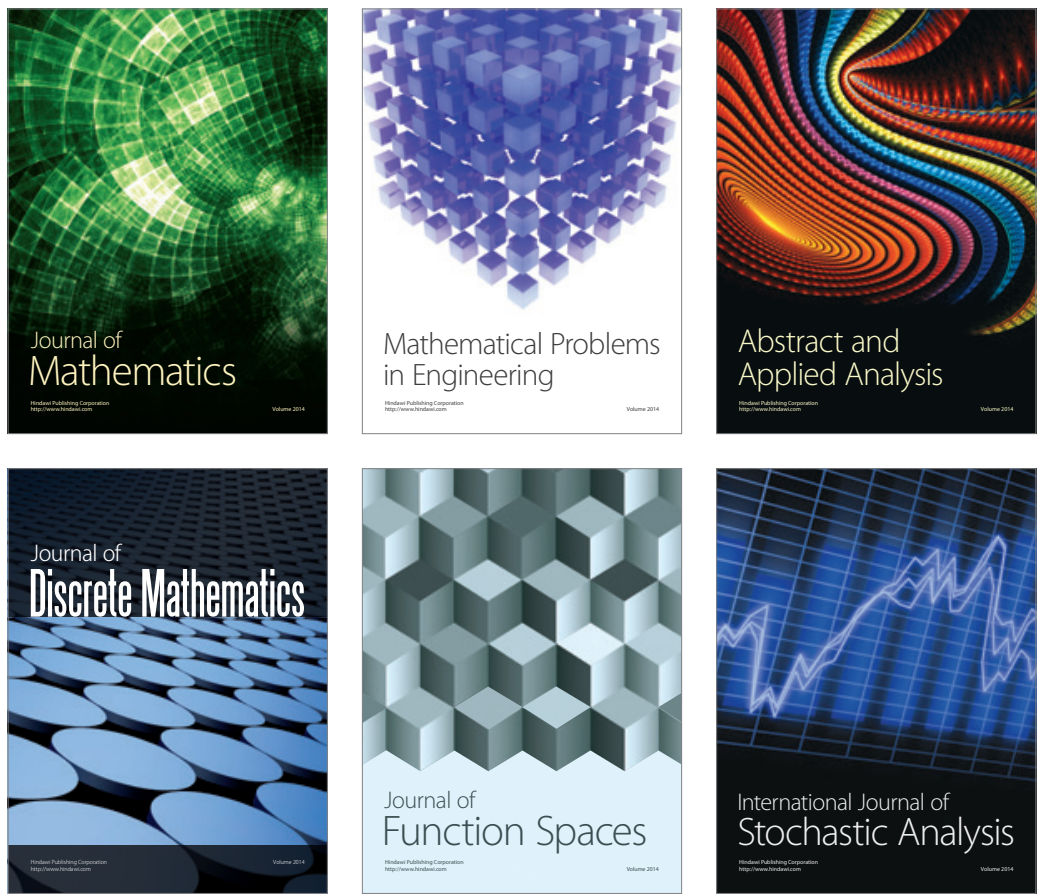

Journal of

Function Spaces

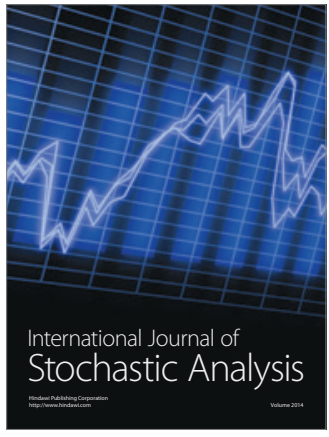

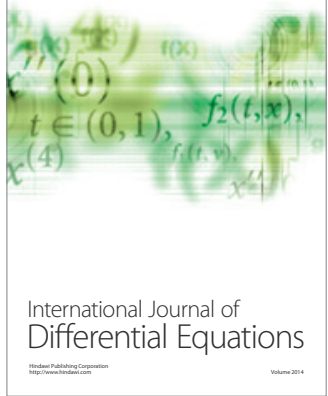
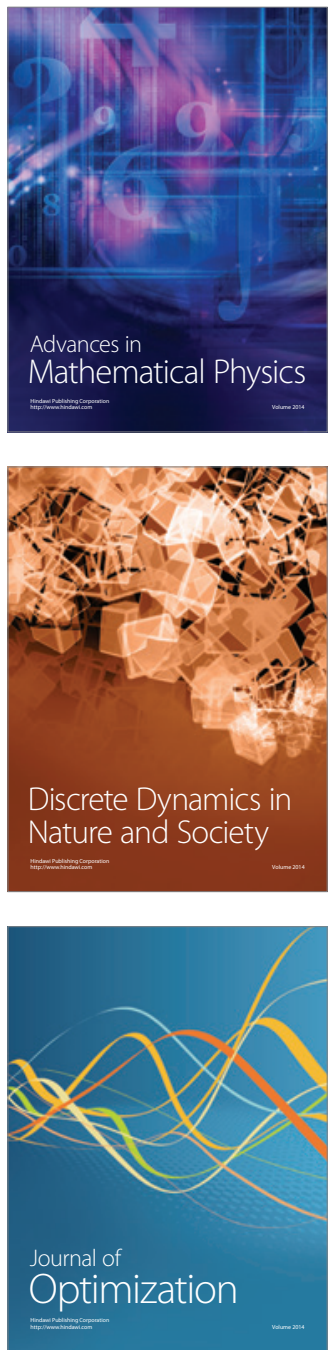